eingeräumt hatte, nicht immer auf die Exemplare beziehen würden, die die ernsten Schäden verursacht hätten, weil der Wolf ein Tier sei, das im Allgemeinen im Rudel lebe. ${ }^{49}$ Der EuGH stellte jedoch fest, dass dann ausreichend dargelegt werden müsse, dass das Schießen irgendeines Wolfes zur Verhütung ernster Schäden im Sinne von Art. 16 Abs. 1 Buchst. b der FFH-Richtlinie geeignet sei. Ansonsten würde gegen die Verpflichtungen aus Art. 12 Abs. 1 und Art. 16 Abs. 1 Buchst. b der FFH-Richtlinie verstoßen. ${ }^{50}$

Ein solches Verständnis ergibt sich auch aus dem Sinn und Zweck des EU-Artenschutzrechtes. Aus artenschutzrechtlicher Sicht ist es irrelevant, ob Wolf A oder Wolf B geschossen wird. Entscheidend ist, dass durch die Ausnahme - das Vergrämen, bzw. Töten des Wolfes - das durch sie verfolgte Ziel erreicht werden kann.

\section{Fazit}

Der Erlass der Brandenburgischen Wolfsverordnung ist lobenswert. Die Angst vor dem Wolf muss nicht nur wegen der Nutztierhalter, sondern auch um seiner selbst willen ${ }^{51}$ ernst genommen werden, unabhängig davon, ob sie gerechtfertigt ist oder nicht. Klare rechtliche Regelungen ermöglichen einen Umgang mit dem Wolf, der sich nicht in Emotionalitäten verfängt, sondern sich an nachprüfbaren Fakten orientiert.

$§ 4$ Abs. 1 BbgWolfV bestimmt, dass die Ausnahme ,zur Abwendung erheblicher wirtschaftlicher Schäden" besteht. $§ 4$ Abs. 2 BbgWolfV regelt, in welchen Fällen von einem solchen Schaden auszugehen ist. Mit Blick auf das hohe Schutzniveau ist ein erheblicher wirtschaftlicher Schaden erst dann anzunehmen, wenn ein einzelner Betrieb in einer grundrechtsrelevanten Weise betroffen ist, die es ihm nicht länger ermöglicht, den Betrieb ohne schwere Einschränkungen fortzuführen.

Die Tatbestandsvoraussetzungen der geeigneten Alternativen und des Sicherstellens des Erhaltungszustandes werden von dem Verordnungstext aufgenommen. Gelungen erscheint insbesondere die Aufnahme der Mindestanforderungen an den Herdenschutz in die Verordnung als Anlage $\mathrm{zu} \ 4$ BbgWolfV.

Es bleibt vor allem zu hoffen, dass die Wolfsverordnung ein weiterer Schritt dahin ist, die Debatte um die Rückkehr des Wolfes zu entemotionalisieren. Wichtig ist eine rechtliche Regelung, die die völker-, europa- und nationalen Vorgaben in ausreichendem Maße beachtet, und gleichzeitig Schutz vor erheblichen wirtschaftlichen Schäden bietet. Die Verordnung macht Wolf und Lamm nicht zu Freunden, aber stellt den Versuch an, beide sicher schlafen zu lassen.

49) EuGH, Urt. v. 14.6.2007 - C-342/05, ECLI:EU:C:2007:341, NuR 2007, S. 477, 479, Rdnr. 41.

50) EuGH, Urt. v. 14.6.2007 - C-342/05, ECLI:EU:C:2007:341, NuR 2007, S. 477, 479, Rdnr. 47.

51) $14 \%$ aller Totfunde beruhen laut Landesamt für Umwelt (LfU) auf illegalen Tötungen, siehe https://lfu.brandenburg.de/cms/ detail.php/bb1.c.411113.de, abgerufen am 26.7.18.

\title{
Rechtliche Aspekte der Anwendung des pharmakologisch wirksamen Stoffs Tamoxifen bei der konditionellen Genausschaltung im Versuchstier Maus
} Justyna Chmielewska, Bettina Bert, Barbara Grune, Gilbert Schönfelder

(c) Der/die Autor(en) 2019. Dieser Artikel ist eine Open-Access-Publikation.

Bei dem Einsatz von pharmakologisch wirksamen Substanzen treffen das Tierversuchsrecht und das Arzneimittelrecht aufeinander. Dieser Beitrag zeigt die in der Tierversuchspraxis in diesem Kontext auftretenden Probleme auf und schlägt praxistangliche Lösungsansätze vor.

\section{Einleitung}

Im Zusammenhang mit Tierversuchen werden aus verschiedenen Gründen pharmakologisch wirksame Stoffe Tieren verabreicht.

\footnotetext{
Ass. jur. Justyna Chmielewska, PD Dr. med. vet. Bettina Bert, Dr. med. vet. Barbara Grune,

Bundesinstitut für Risikobewertung, Nationaler Ausschuss für den Schutz von für wissenschaftliche Zwecke verwendeten Tieren, Berlin, Deutschland

Univ.-Prof. Dr. med. Gilbert Schönfelder, Bundesinstitut für Risikobewertung, Nationaler Ausschuss für den Schutz von für wissenschaftliche Zwecke verwendeten Tieren; Charité-Universitätsmedizin,

Institut für Klinische Pharmakologie und Toxikologie, Berlin, Deutschland
}

Zum ersten werden, wie bei allen anderen Tieren, auch bei Versuchstieren Arzneimittel zur Behandlung oder Vorbeugung einer Erkrankung bzw. zur Durchführung einer Narkose oder Analgesie angewendet. Dabei handelt es sich um die klassische Anwendung nach tierärztlicher Indikation, wofür in der Regel zugelassene Arzneimittel verwendet werden. Die Zulässigkeit der Anwendung richtet sich zweifelsohne nach dem Arzneimittelgesetz, sodass diesbezüglich eine gesicherte Rechtslage vorhanden ist.

Ein zweiter Anwendungsfall ist die Verabreichung von pharmakologisch wirksamen Stoffen bei Versuchstieren innerhalb des Zulassungsverfahrens eines Arzneimittels, um dessen Wirkung zu untersuchen. Dies geschieht insbesondere im Rahmen der gesetzlich vorgeschriebenen Tierversuche zur Entwicklung, Herstellung und Prüfung der Qualität, Wirksamkeit und Unbedenklichkeit von Arzneimitteln zur Anwendung beim Menschen oder beim Tier ( $\$ 7$ a Abs. 1 Nr. 4 TierSchG). Hier werden bei Versuchstieren pharmakologisch wirksame Stoffe eingesetzt, die keine zugelassenen Arzneimittel sind. Die Zulässigkeit der Anwendung solcher Substanzen ergibt sich aus der Natur der Sache: Es handelt sich um Tierversuche, die gem. \$7a Abs. 1 Nr. 4 TierSchG der Testung von Substanzen dienen: Die Ergebnisse solcher Versuche sind gem. $\$ 22$ Abs. 2 Nr. 2 
AMG im Rahmen des Zulassungsverfahrens vorzulegen, daher bestehen auch keine Zweifel an der Zulässigkeit der Anwendung dieser Substanzen bei Versuchstieren.

Darüber hinaus werden pharmakologisch wirksame Stoffe aber auch bei Versuchstieren zu weiteren Forschungszwecken angewendet, um einen bestimmten biologischen Effekt zu erzielen. Ein Beispiel für eine solche Anwendung ist die Herstellung gentechnisch veränderter Organismen. Mäuse sind noch immer die am häufigsten verwendeten Versuchstiere in der biomedizinischen Forschung, ${ }^{1}$ insbesondere weil sie einfach genetisch zu manipulieren sind, um Krankheitsmodelle zu entwickeln. Die Verabreichung der pharmakologisch wirksamen Stoffe Tamoxifen und Tetracyclin wird genutzt, um gezielt die Expression von Genen zu einem bestimmten Zeitpunkt bei den Versuchstieren zu regulieren. Diese Vorgehensweise wird angewandt, um das Tiermodell für weitere Untersuchungen, wie z.B. Beobachtungen der Änderung ihres Phänotyps, zu nutzen. Es besteht jedoch Unklarheit darüber, ob das Verabreichen von Tamoxifen oder anderen pharmakologisch wirksamen Stoffen unter den Tatbestand eines Tierversuches i.S. v. $\$ 7$ Abs. 2 TierSchG zu subsumieren ist und ob die Vorschriften des AMG in diesem Fall der Anwendung dieser Substanzen bei Versuchstieren einschlägig sind.

Auf der Suche nach neuen Medikamenten zur Behandlung ,hormon-aktiver“ Tumore wurde in den 1960iger und 1970iger Jahren Tamoxifen als neues Medikament zur Behandlung von Brustkrebs erstmals in Großbritannien eingeführt. $^{2}$ Tamoxifen wird als zugelassenes Arzneimittel bei Menschen in der Therapie von Brustdrüsentumoren auch in Deutschland eingesetzt. Vereinfacht dargestellt beruht die Wirkung von Tamoxifen darauf, dass bei der oralen Verabreichung von Tamoxifen durch die Verstoffwechselung im Körper zwei wichtige biologisch aktive Metaboliten (Abbauprodukte) 4-Hydroxy-Tamoxifen und Endoxifen (4-Hydroxy-N-Desmethyl-Tamoxifen) entstehen. Tamoxifen (eher schwach wirksam), 4-Hydroxy-Tamoxifen und Endoxifen binden kompetitiv zu den endogenen Östrogenen an dem zytoplasmatischen Östrogenrezeptor. Dies führt zu einer Abnahme der Zellteilung in östrogenabhängigen Geweben, was die Abnahme der Proliferation östrogenabhängigen Gewebes bewirkt und das weitere Tumorwachstum bzw. Rezidive verhindert. ${ }^{3}$

In der tierexperimentellen Forschung nutzte man das Wissen über die pharmakologische Wirkung von Tamoxifen bei der Entwicklung transgener Mausmodelle speziell zum Zweck konditioneller genommodifizierender Eingriffe bei der Maus. Dafür werden sog. induzierbare Cre/ LoxP-Rekombinationssysteme verwendet. Im entsprechenden gentechnisch modifizierten Mausmodell wird ein Fusionsprotein bestehend aus der modifizierten LigandenBindungsdomäne des Östrogenrezeptors und der Cre-Rekombinase exprimiert. ${ }^{4}$ Nach Verabreichung von Tamoxifen bzw. seines aktiven Metaboliten 4-Hydroxytamoxifen (4-OHT) binden diese an der modifizierten Liganden-Bindungsdomäne des Fusionsproteins. Die fusionierte Cre-Rekombinase verlagert sich vom Zytoplasma in den Zellkern. Dort erkennt es die in das Genom der Maus integrierte Sequenz loxP und schneidet die zwischen zwei loxP-Seiten befindliche DNA aus dem Genom und erzielt so den gewünschten Effekt. ${ }^{5}$ Damit ist es möglich, durch Gabe von Tamoxifen Gene gezielt in verschiedenen Geweben und zu definierten Zeitpunkten auszuschalten und so Tiermodelle zur Untersuchung bestimmter Krankheiten und Pathomechanismen zu schaffen. Die Verabreichung von Tamoxifen bezweckt in diesem Fall die Herbeiführung der konditionellen Genausschaltung. Sie verfolgt weder ein therapeutisches Ziel, noch dient sie der Überprüfung der Wirksamkeit von Tamoxifen als Substanz.

Tamoxifen ist als zugelassenes Humanarzneimittel in Tablettenform erhältlich. Hier liegt der pharmakologisch wirksame Stoff als Tamoxifencitrat vor. Tamoxifen ist auch als freie Base im chemischen, biochemischen und pharmazeutischen Fachhandel erhältlich. Beide Substanzen (Tamoxifencitrat und Tamoxifen freie Base) können auf unterschiedlichem Weg (oral, subkutan und intraperitoneal (i. p.)) der Maus appliziert werden, sind nach entsprechender galenischer Aufbereitung bioverfügbar ${ }^{6}$ und können so ihre pharmakologische Wirkung entfalten. Das zurzeit gängigste Vorgehen zur Verabreichung von Tamoxifen bei Mäusen ist die intraperitoneale Injektion einer eigens dafür aus der Tamoxifen-Base und einer Trägersubstanz hergestellten öligen Suspension. Hierfür ist es jedoch notwendig, dass die wissenschaftlichen Einrichtungen Tamoxifen als reine Laborsubstanz beziehen, um daraus eine entsprechende Suspension herzustellen.

Dieses Vorgehen ruft vielfältige rechtliche Fragen hervor: Zum einen ist zu prüfen, ob diese ,,vorbereitende Behandlung" den Tatbestand eines Tierversuches i.S.d. Tierschutzgesetzes erfüllt. Zum anderen ist $\mathrm{zu}$ diskutieren, welche Relevanz die Vorschriften des Arzneimittelrechts für diesen Sachverhalt haben.

\section{Rechtliche Einstufung der vorbereitenden Behandlung von Versuchstieren mit arzneilich wirksamen Substanzen, um eine gentechnische Modifikation $\mathrm{zu}$ induzieren}

\subsection{Tierversuch gem. $\int 7$ Abs. 2 S. 1 Nr. 3 TierSchG}

Da die Anwendung von Tamoxifen eine genetische Modifikation herbeiführt, ist zunächst $\$ 7$ Abs. $2 \mathrm{~S}$. 1 Nr. 3 TierSchG zu prüfen, da sich diese Vorschrift explizit auf Veränderungen des Erbguts bezieht.

\subsubsection{Eingriff oder Behandlung am Erbgut von Tieren}

Ein Tierversuch i.S.d. $\$ 7$ Abs. 2 TierSchG setzt zunächst einen Eingriff oder eine Behandlung voraus. Da sich diese Handlung in diesem Fall nicht auf das Tier als solches sondern auf das Erbgut von Tieren bezieht, ist die Definition von Eingriff oder Behandlung dahingehend entsprechend $\mathrm{zu}$ modifizieren, dass eine Manipulation stattfinden muss, mit der gezielt eine Modifikation des Genoms herbeigeführt werden soll. ${ }^{7}$

Tamoxifen wird in der Gentechnik dazu eingesetzt, um mit Hilfe des Cre/Lox-Systems bei Tieren Gene gewebsspezifisch und zu definierten Zeitpunkten an- bzw. abzu-

1) https://www.bmel.de/DE/Tier/Tierschutz/_texte/TierschutzTierforschung.html?docId=10323474\#doc10323474bodyText1.

2) Jordan, Tamoxifen: A most unlikely pioneering medicine, Nature Reviews 2003, S. 205 ff., https://doi.org/10.1038/nrd1031.

3) Fachinformation Tamoxifen $20 \mathrm{mgTabletten,abrufbarunter:http://}$ www.abz.de/index.php?eID $=$ dumpFile $\& \mathrm{t}=\mathrm{f} \& \mathrm{f}=40091 \& \mathrm{~g}=$ $1 \& \mathrm{r}=1872 \% 2 \mathrm{C} 1872 \&$ token $=\mathrm{e} 7 \mathrm{~b} 685 \mathrm{~d} 502 \mathrm{a} 40 \mathrm{aac} 35 \mathrm{fbe} 8 \mathrm{dac} 657 \mathrm{bb}$ b8b41178b9.

4) Zu den Details siehe Metzger u. a., Conditional site-specific recombination in mammalian cells using a ligand-dependent chimeric Cre-recombinase, PNAS 1995, S. $6991 \mathrm{ff}$.

5) Zu den Details des Verfahrens siehe z.B. Metzger/Chambon, Site and Time-Specific Gene Targeting in the Mouse, Methods 24, 71-80 (2001); Hayashi/McMahon, Efficient Recombination in Diverse Tissues by a Tamoxifen-Inducible Form of Cre: A Tool for Temporally Regulated Gene Activation/Inactivation in the Mouse, Developmental Biology 244, 305-318 (2002).

6) Andersson u.a., Tamoxifen administration routes and dosage for inducible Cre-mediated gene disruption in mouse hearts, Transgenic Res 2010, S. 715, 718 ff.; Whitfield u. a., Tamoxifen administration to mice, Cold Spring Harb Protoc, 2015, S. $269 \mathrm{ff}$., https:// doi.org/10.1101/pdb.prot077966; Harper/Walpole, Contrasting endocrine activities of cis and trans isomers in a series of substituted triphenylethylenes, Nature 1996, S. 87.

7) $\mathrm{Zu}$ der herkömmlichen Definition des Eingriffes bzw. der Behandlung Hirt/Maisack/Moritz, Tierschutzgesetz, 3. Aufl. 2016, \7 TierSchG, Rdnr. 6. 
schalten. Damit bewirkt die durchgeführte Handlung in der Tat eine Veränderung im Genom des betroffenen Tieres. Gleichwohl kann hier angezweifelt werden, ob die reine Applikation von Tamoxifen tatsächlich mit einer Manipulation ,,am Erbgut" von Tieren gleichzusetzen ist. \$7 Abs. 2 S. 1 Nr. 3 TierSchG geht auf das Gentechnikgesetz vom 20.6.1990 (BGB1. I S. 1080, Art. 5) zurück. Das Ziel dieser Regelung war, Versuche am Erbgut zum Schutz der davon betroffenen Tiere zu beschränken. ${ }^{8}$ Bezweckt wurde mit dieser Regelung, dass auch diejenigen Fälle unter den Begriff des Tierversuchs subsumiert werden können, bei denen die Manipulationen zu Versuchszwecken statt am lebenden Tier an Eizellen oder Embryonen vorgenommen wird und die Möglichkeit besteht, dass dadurch entweder das später entstehende Tier oder das Trägertier Schmerzen, Leiden oder Schäden erfährt. ${ }^{9}$ Dies ergibt sich auch aus der systematischen Gegenüberstellung der verschiedenen Regelungsalternativen des $\$ 7$ Abs. 2 S. 1 TierSchG: Während von Nr. 1 alle Varianten des klassischen experimentellen Versuchs umfasst sind, bei welchen ein Eingriff oder eine Behandlung direkt am Tier vorgenommen wird, decken die Regelungen der Nr. 2 und 3 die etwas weniger typischen Fälle eines Tierversuchs ab: Zum einen den Fall der Zucht von Tieren, bei der die Nachkommen Schmerzen, Leiden oder Schäden erfahren können, zum anderen den Fall, in welchem eine genetische Manipulation am Erbgut, d. h. zunächst in Zellen außerhalb des Tierorganismus, stattfindet, wodurch jedoch Tiere erzeugt werden können, die ebenfalls Schmerzen, Leiden oder Schäden erleiden können.

\subsubsection{Zwischenergebnis}

Damit fällt die Induktion eines Gen-Knockouts durch Gabe von Tamoxifen oder anderen chemischen Substanzen nicht unter den Tatbestand des $₫ 7$ Abs. 2 S. 1 Nr. 3 TierSchG.

\subsection{Tierversuch gem. $\int 7$ Abs. 2 S. 1 Nr. 1 TierSch G}

Wie oben schon erwähnt, umfasst der Tatbestand des $₫ 7$ Abs. 2 S. 1 Nr. 1 TierSchG den klassischen experimentellen Tierversuch. Dieser setzt voraus, dass am Tier ein Eingriff oder eine Behandlung zu Versuchszwecken stattfinden, die zur Konsequenz haben kann, dass das Tier Schmerzen, Leiden oder Schäden erfährt.

\subsubsection{Eingriff oder Behandlung}

Zunächst müsste es sich bei der Gabe von Tamoxifen um einen Eingriff oder eine Behandlung handeln. Unter Eingriffen werden Maßnahmen verstanden, die zu einer Störung der körperlichen Unversehrtheit führen oder physiologische Abläufe auf Zeit oder auf Dauer verändern. ${ }^{10}$ Durch eine i.p.-Injektion wird die Bauchhöhle perforiert und eine Substanz injiziert. Dadurch wird die körperliche Unversehrtheit des Tieres beeinträchtigt, sodass ein Eingriff i.S. d. Tierschutzgesetzes vorliegt.

Im Falle der Tamoxifen-Gabe über das Futter liegt kein Eingriff vor, da keine invasive Handlung durchgeführt wird. Dennoch könnte es sich um eine Behandlung i.S.d. \7 Abs. 2 S. 1 Nr. 1 TierSchG handeln. Unter Behandlungen sind Beeinträchtigungen der körperlichen Integrität zu verstehen, die noch nicht die Schwelle zum Eingriff erreichen. Diese können direkt oder indirekt sein, als Beispiel werden in der Literatur u. a. Fütterungsversuche genannt. ${ }^{11}$ Mit der Gabe einer Substanz im Futter wird diese vom Tier aufgenommen und der Körper den entsprechenden Wirkungen ausgesetzt. Damit ist eine Behandlung i.S.d. Tierschutzgesetzes zu bejahen.

\subsubsection{Zu Versuchszwecken}

$\mathrm{Zu}$ Versuchszwecken wird eine Maßnahme vorgenommen, wenn mit ihr eine wissenschaftliche Fragestellung, die noch nicht hinreichend geklärt ist, beantwortet werden soll. ${ }^{12}$
Die Gabe von Tamoxifen bewirkt, dass ein Gen ausgeschaltet wird, damit das Tier den für das spätere experimentelle Vorgehen anvisierten Phänotyp zeigt. Das bedeutet, dass es bei der Gabe von Tamoxifen nicht darum geht, die eigentliche Wirkung dieses pharmazeutischen Wirkstoffes zu untersuchen, sondern dass Tamoxifen bei diesen Versuchen lediglich als Werkzeug dient. Dennoch ist die Gabe von Tamoxifen Teil einer wissenschaftlichen Fragestellung und trägt dazu bei, neue Erkenntnisse zu erzielen. Die Induktion einer genetischen Modifikation ist der erste Schritt im Ablauf des gesamten Experiments, die als „Vorbereitungshandlung“" nicht von dem ,eigentlichen“" Versuch getrennt betrachtet werden kann. Die Gabe von Tamoxifen und die darauffolgenden Untersuchungen, seien es Untersuchungen von Gewebe am getöteten Tier oder die Durchführung von weiteren Tests oder Behandlungen an lebenden Tieren, bilden vielmehr eine Einheit. Die Situation, dass Versuchstiere durch einen bestimmten Eingriff oder Behandlung auf die eigentliche ,experimentelle“ Handlung vorbereitet werden, kommt häufig vor, z. B. bei der Gabe von bestimmten Substanzen vor Verhaltensversuchen oder eine stereotaktische Operation. Ähnlich verhält es sich auch mit der Gabe von Tamoxifen. Das Auslösen eines Gen-Knockouts ist die Grundlage für weiteres wissenschaftliches Vorgehen im Rahmen eines Tierversuches und bildet mit ihm eine logische und tatsächliche Einheit. Für diese Einschätzung spricht ebenfalls, dass im Falle des $\$ 7$ Abs. 2 S. 1 Nr. 3 TierSchG alle vorbereitenden Eingriffe, die an lebenden Tieren vorgenommen werden, die potentiell belastend sind (etwa Erzeugung der Superovulation, Entnahme der Eizellen, Vasektomie bei männlichen Tieren, Einsetzen der manipulierten Eizellen beim Muttertier) als Tierversuch gesehen werden, und zwar sowohl i.S.d. $\$ 7$ Abs. 2 S. 1 Nr. 3 als auch Nr. 1 TierSchG. ${ }^{13}$

\subsubsection{Möglichkeit des Auftretens von Schmerzen, Leiden} oder Schäden beim betroffenen Tier

Die Gabe von Tamoxifen mittels einer i.p.-Injektion ist schon an sich ein invasiver Eingriff, der Schmerzen verursachen kann, ebenso sind durch die Wirkung von Tamoxifen auf den Körper des Tieres Schmerzen, Leiden oder Schäden nicht ausgeschlossen. Die Gabe von Tamoxifen über das Futter verursacht zwar als solche keine Schmerzen, Leiden oder Schäden. Aufgrund der Wirkung des pharmazeutischen Wirkstoffes auf den Körper des Tieres können jedoch trotzdem unerwünschte Wirkungen auftreten. ${ }^{14}$

Darüber hinaus kann das Tier weitere Schmerzen, Leiden oder Schäden erfahren, je nachdem mit welchen physiologischen und biochemischen Konsequenzen der mittels Tamoxifen induzierte Phänotyp einhergeht. Darüber hinaus ist die Möglichkeit des Auftretens von Schmerzen, Leiden oder Schäden im Hinblick auf das gesamte Versuchsgesche-

8) Lorz/Metzger, Tierschutzgesetz, 6. Aufl. 2008, $\$ 7$ TierSchG, Rdnr. $10 \mathrm{ff}$

9) Hirt/Maisack/Moritz, Tierschutzgesetz, 3. Aufl. 2016, $\$ 7$ TierSchG, Rdnr. 8

10) Hirt/Maisack/Moritz, Tierschutzgesetz, 3. Aufl. 2016, $\$ 5$ TierSchG, Rdnr. 5.

11) Hirt/Maisack/Moritz, Tierschutzgesetz, 3. Aufl. 2016, $\$ 7$ TierSchG, Rdnr. 6

12) Hirt/Maisack/Moritz, Tierschutzgesetz, 3. Aufl. 2016, $\$ 7$ TierSchG, Rdnr. 6.

13) Hirt/Maisack/Moritz, Tierschutzgesetz, 3. Aufl. 2016, $\$ 7$ TierSchG, Rdnr. 8 .

14) In der Literatur wird z.B. von (z.T. vorübergehendem) Gewichtsverlust berichtet: Andersson u.a., Tamoxifen administration routes and dosage for inducible Cre-mediated gene disruption in mouse hearts, Transgenic Res 2010, 715, 721 f.; Kiermeyer u.a., Optimization of Spaciotemporal Gene Inactivation in Mouse Heart by Oral Application of Tamoxifen Citrate, genesis $2007,11,12$ 
hen zu beurteilen, die Gabe von Tamoxifen ist, wie oben dargelegt, nur ein Teil davon.

\subsection{Ergebnis}

Damit ist die Behandlung von Versuchstieren mit Substanzen wie Tamoxifen, um einen bestimmten Phänotyp zu induzieren, als Tierversuch i.S.d. $\$ 7$ Abs. 2 S. 1 Nr. 1 TierSchG anzusehen.

\section{Anwendbarkeit der arzneimittelrechtlichen Vorschriften}

Im Zusammenhang mit dem Einsatz von pharmazeutischen Wirkstoffen bei Versuchstieren werden jedoch weitere rechtliche Probleme aufgeworfen. In der Praxis stellen sich die Anwender oft die Frage, ob der Einsatz von Tamoxifen in der oben beschriebenen Konstellation den Vorschriften des Arzneimittelrechts unterliegt. Die Zweifel wurzeln darin, dass die Substanz Tamoxifen auch als Humanarzneimittel auf dem Markt existiert. Daher ist eine Klärung des Verhältnisses zwischen dem Tierversuchsrecht und dem Arzneimittelrecht notwendig.

\81 AMG trifft eine Regelung über das Verhältnis des Arzneimittelgesetzes zu anderen Rechtsgebieten, mit welchen es Uberschneidungen gibt. Demnach bleiben die Vorschriften des Tierschutzgesetzes unberührt. Mit dieser Formulierung kann Verschiedenes zum Ausdruck gebracht werden: wenn sich die Anwendungsbereiche nicht überschneiden, kann es sich dabei um einen klarstellenden Hinweis auf andere Rechtsnormen handeln. Diese Formulierung kann jedoch auch das Gebot ausdrücken, dass beide Rechtsgebiete nebeneinander angewendet werden sollen oder dass ein bestimmtes Rangverhältnis besteht. ${ }^{15}$

Aus der Regelung des $₫ 81$ AMG ergibt sich jedenfalls zunächst, dass die dort genannten bundesrechtlichen Regelungen nicht vom AMG beschränkt oder verdrängt werden sollen, sondern gehen dem AMG vor, falls sie im Verhältnis zum AMG widersprüchliche Inhalte enthalten ${ }^{16}$ bzw. neben dem AMG Anwendung finden. ${ }^{17}$

Die Nennung des Tierschutzgesetzes in dieser Vorschrift erfolgte jedoch insbesondere im Hinblick auf die pharmakologisch-toxikologischen Prüfungen von Arzneimitteln, deren Vorlage im Rahmen des Zulassungsantrags nach $\$ 22$ Abs. 2 Nr. 2 AMG vorgeschrieben ist, sowie die klinischen Prüfungen von Arzneimitteln bei Tieren nach \$59 AMG. Mit der Regelung des $\int 81$ AMG wollte der Gesetzgeber sicherstellen, dass auch im Rahmen der o. g. gesetzlich geforderten Prüfungen die tierschutzrechtlichen Belange Beachtung finden..$^{18}$

Da die Kollisionsregelung keine Antwort auf die hier relevante Frage liefern kann, ist deshalb zu prüfen, ob im Falle der Anwendung von Tamoxifen als gentechnisches Werkzeug bei Versuchstieren der Tatbestand des $\$ 1$ AMG erfüllt ist und damit der Anwendungsbereich des Arzneimittelgesetzes eröffnet ist.

\subsection{Arzneimitteleigenschaft}

Das Arzneimittelgesetz kommt gem. \$S 1, 2 AMG nur dann zur Anwendung, wenn es sich bei der fraglichen Substanz um ein Arzneimittel handelt. So ist zu prüfen, ob Substanzen wie Tamoxifen, die zur Induktion eines Phänotyps benutzt werden, in dieser konkreten Art ihrer Anwendung als Arzneimittel i. S. d. AMG anzusehen sind.

\subsubsection{Begriff des Arzneimittels im Sinne} des Arzneimittelgesetzes

Das Arzneimittelgesetz unterscheidet im $₫ 2$ Abs. 1 zwischen sog. Präsentationsarzneimitteln $(\$ 2$ Abs. 1 Nr. 1 AMG) und den Funktionsarzneimitteln ( $\$ 2$ Abs. 1 Nr. 2 AMG). Darüber hinaus gelten gem. $\$ 2$ Abs. 2 AMG be- stimmte Gegenstände als Arzneimittel, obwohl sie die Definition des $\$ 2$ Abs. 1 AMG nicht erfüllen, sog. fiktive Arzneimittel. Für die Bestimmung, ob die Anwendung von Tamoxifen als ,gentechnisches Werkzeug“ in den Anwendungsbereich des AMG fällt ist entscheidend, ob Tamoxifen in der Form einer reinen Laborchemikalie die Definition eines Arzneimittels i.S. d. AMG erfüllt. Diese Prüfung soll sich explizit auf Tamoxifen in Form einer Laborchemikalie und die daraus durch die Wissenschaftler hergestellte Injektionslösung bzw. Futtermittel beziehen, da Tamoxifen zumeist in diesen Formen bei Versuchstieren zur Anwendung kommt. Es kommt hingegen nicht darauf an, ob die für den humanen Gebrauch bestimmten Tamoxifen-Tabletten in diesem Fall als Arzneimittel anzusehen sind.

$\$ 2$ Abs. 3 AMG schließt bestimmte Erzeugnisse aus dem Anwendungsbereich des AMG aus. Die hier relevante Laborchemikalie Tamoxifen fällt jedoch eindeutig nicht unter diesen Tatbestand, da es sich hier offensichtlich nicht um Lebensmittel, kosmetische Mittel, Tabakerzeugnisse bzw. andere im $\$ 2$ Abs. 3 AMG aufgelistete Produkte handelt.

\subsubsection{Präsentationsarzneimittel nach $\$ 2$ Abs. $1 \mathrm{Nr}$. 1 AMG}

Zunächst muss sich bei einem Arzneimittel um einen Stoff oder eine Zubereitung aus Stoffen handeln. Was unter dem Begriff eines Stoffes zu verstehen ist, ist im $\$ 3$ AMG legal definiert. Demnach sind gem. $\$ 3$ Nr. 1 AMG Stoffe chemische Elemente und chemische Verbindungen sowie deren natürlich vorkommende Gemische und Lösungen. Sowohl Tamoxifen als auch andere Substanzen, deren Verwendung als „gentechnisches Werkzeug" denkbar ist, sind in jedem Fall chemische Verbindungen und damit Stoffe i. S.d. Arzneimittelgesetzes.

Die aus der Reinsubstanz hergestellte Lösung wird entweder in den Körper von Mäusen injiziert oder die Tiere werden mit einem tamoxifenhaltigen Futtermittel gefüttert, um einen bestimmten Phänotyp zu erzeugen. Damit liegt auch eine Anwendung im tierischen Körper gem. $₫ 2$ Abs. 1 Nr. 1 AMG vor.

Des Weiteren muss es sich bei Präsentationsarzneimitteln um Produkte handeln, die als Mittel mit Eigenschaften zur Heilung, Linderung oder Verhütung von Krankheiten oder krankhaften Beschwerden bestimmt sind. Entscheidend ist dabei, dass die jeweiligen Produkte objektiv oder subjektiv dazu geeignet sind, den therapeutischen bzw. prophylaktischen Zwecken zu dienen. ${ }^{19}$

Bei dem hier relevanten Produkt handelt es sich um Tamoxifen als Laborchemikalie. Diese Chemikalie an sich ist nicht auf die Art und Weise verarbeitet, die notwendig wäre, um sie zur Behandlung oder Prophylaxe von Krankheiten einzusetzen. Dazu müsste dieser Stoff zunächst in eine entsprechende Darreichungsform in einer entsprechenden Konzentration gebracht werden. Daher eignet sich diese Chemikalie zunächst nur für den Einsatz im Labor, um bestimmte Effekte im Rahmen von Versuchen hervorzurufen oder Untersuchungen durchzuführen.

Damit handelt es sich in dieser Form um kein Mittel mit Eigenschaften zur Heilung, Linderung oder Verhütung von menschlichen oder tierischen Krankheiten.

So ist die Laborchemikalie kein Präsentationsarzneimittel i.S.d. $\$ 2$ Abs. 1 Nr. 1 AMG.

15) Handbuch der Rechtsförmlichkeit, BMJV, S. 42 f. http://www. bmjv.de/SharedDocs/Downloads/DE/PDF/Themenseiten/ RechtssetzungBuerokratieabbau/HandbuchDerRechtsfoermlichkeit_deu.pdf?__blob=publicationFile

16) Nickel in: Kügel/Müller/Hofmann, Arzneimittelgesetz, 2. Aufl. 2016, \$81 AMG, Rdnr. 2.

17) Rehmann, Arzneimittelgesetz, 4. Aufl. 2014, \$81 AMG, Rdnr. 1.

18) Nickel in: Kügel/Müller/Hofmann, Arzneimittelgesetz, 2. Aufl. 2016, \$81 AMG, Rdnr. 5.

19) Kügel/Müller/Hoffmann, Arzneimittelgesetz, 2. Aufl. 2016, $\mathbb{} 2$ AMG, Rdnr. 80. 


\subsubsection{Funktionsarzneimittel gem. $\$ 2$ Abs. 1 Nr. 2 AMG}

Es könnte sich jedoch um ein Funktionsarzneimittel handeln. Ähnlich wie beim Begriff des Präsentationsarzneimittels muss es sich bei einem Funktionsarzneimittel um Stoffe oder Zubereitungen aus Stoffen handeln, die wiederum im oder am menschlichen oder tierischen Körper angewendet werden können. Wie oben bereits festgestellt, ist diese Voraussetzung erfüllt.

Ein Funktionsarzneimittel i. S. d. $\$ 2$ Abs. 1 Nr. 2 Buchst. a AMG muss darüber hinaus eins der in dieser Vorschrift genannten Ziele verfolgen, d.h. physiologische Funktionen durch eine pharmakologische, immunologische oder metabolische Wirkung wiederherzustellen, zu korrigieren oder $\mathrm{zu}$ beeinflussen.

$\mathrm{Zu}$ prüfen ist, ob die Verabreichung von Tamoxifen, um einen Gen-Knockout herbeizuführen, mit einem der o. g. Ziele erfolgt.

Unter physiologischen Funktionen sind in diesem Kontext die natürlichen Lebensvorgänge zu verstehen. Es sind alle Vorgänge und Aufgaben, die der menschliche oder tierische Körper in seiner Gesamtheit oder durch einzelne Körperteile oder Organe verrichtet. Als Beispiele für physiologische Funktionen werden das Wachstum, die Regeneration, die Atmung sowie die Aktivität des Nervensystems, des Herz-Kreislaufsystems und des Stoffwechsels genannt. $^{20}$

Es ist fraglich, ob bei Tamoxifengabe zur Induktion eines Gen-Knockouts eine Einwirkung auf die physiologischen Funktionen des Körpers überhaupt stattfindet. Mit der Gabe dieser Substanz werden indirekt Veränderungen der DNA bewirkt, d. h. Tamoxifen bindet an einen genetisch modifizierten Östrogenrezeptor, was dazu führt, dass die Cre-Recombinase das Zielgen aus der DNA schneiden kann. Daraus könnte geschlussfolgert werden, dass Tamoxifen als gentechnisches Werkzeug nicht unmittelbar die physiologischen Vorgänge beeinflusst. Eventuelle Veränderungen der physiologischen Vorgänge, die mit dem GenKnockout und der Manifestation des entsprechenden Phänotyps einhergehen, sind erst die Folge der genetischen Modifikation, nicht aber unmittelbar der Gabe von Tamoxifen als solchen.

Andererseits könnte jedoch argumentiert werden, dass der durch Gabe von Tamoxifen induzierte Phänotyp in der Regel Auswirkungen auf physiologische Funktionen mit sich bringt. Aufgrund der so begründeten Kausalität zwischen der Gabe von Tamoxifen und dem Auftreten einer Veränderung von physiologischen Funktionen im Tier könnte eine Beeinflussung physiologischer Funktionen i. S.d. $\$ 2$ Abs. 1 Nr. 2 AMG bejaht werden.

Die Entscheidung darüber, welcher der genannten Auslegungsvarianten Vorzug zu geben ist, erscheint jedoch entbehrlich. Auch wenn der zweiten Auslegungsvariante gefolgt wird und angenommen wird, dass Tamoxifen als gentechnisches Werkzeug physiologische Funktionen beeinflusst, so darf die wesentliche Aufgabe des Stoffes, welcher als Funktionsarzneimittel in Betracht gezogen wird, nicht unberücksichtigt bleiben. ${ }^{21}$ Die Einstufung einer Substanz als Funktionsarzneimittel kann nicht alleine deshalb erfolgen, weil diese Substanz nachteilige Wirkungen auf den Organismus entfaltet. Das AMG sieht schließlich die Funktion eines Arzneimittels in der Therapie von Krankheiten bzw. in der Bekämpfung von unerwünschten körperlichen Zuständen. Dafür spricht auch, dass die Beeinflussung von physiologischen Funktionen in einer Reihe mit den Begriffen „Korrektur“ und „Wiederherstellung" physiologischer Funktionen genannt wird, die eindeutig das Element einer positiven Einwirkung auf den Körper bzw. einer therapeutischen Intention beinhalten. Es ist aus dem Gesamtzusammenhang auch nicht erkennbar, dass das Tatbestandsmerkmal der Beeinflussung physiologischer Funktionen einen anderen Charakter haben soll. Daher spricht die systematische Auslegung dafür, dass nur Produkte mit therapeutischer oder prophylaktischer Zweckbestimmung unter den Begriff des Funktionsarzneimittels subsumiert werden sollen. Von seiner Zweckbestimmung her soll das Arzneimittelgesetz keine Produkte erfassen, mit denen primär andere Zwecke verfolgt werden. ${ }^{22}$ Die Eigenschaft eines Funktionsarzneimittels wurde bisher insbesondere für diverse Ernährungs- oder Genussmittel verneint. ${ }^{23}$ Der Begriff des Funktionsarzneimittels erfüllt zwar gewissermaßen die Funktion eines Auffangtatbestandes zum Begriff des Präsentationsarzneimittels, er darf dennoch nicht zu weit ausgelegt werden. Die physiologischen Funktionen können nämlich durch zahlreiche Substanzen beeinflusst werden, wie z.B. Lebensmittel, Genussmittel oder auch Giftstoffe. All diese Substanzen unter den Begriff des Funktionsarzneimittels zu subsumieren, obwohl sie keinen arzneimittelspezifischen Bezug aufweisen, würde zu nicht mehr sachgerechten Ergebnissen führen. ${ }^{24}$

Diese Grundsätze, die in der oben zitierten Rechtsprechung zur rechtlichen Einstufung von E-Zigaretten erarbeitet wurden, lassen sich gut auf die Situation von der Anwendung von Tamoxifen bei Versuchstieren übertragen. Auch hier findet zwar eine (nachteilige) Beeinflussung der physiologischen Funktionen statt, diese dient jedoch einem bestimmten experimentellen Zweck und hat zu keinem Zeitpunkt die Zielsetzung einer Therapie oder Bekämpfung unerwünschter körperlicher Zustände beim Tier. Genauer gesagt, geht es hier ausschließlich darum, ein geeignetes Modell für die anvisierte wissenschaftliche Fragestellung zu generieren. Das Tier und sein Körper stehen als solche im Moment der Tamoxifengabe nicht einmal im Mittelpunkt.

Die Motivation des Gesetzgebers, die Kategorie des Funktionsarzneimittels einzuführen, war auch Mittel, die keines der Ziele aus $₫ 2$ Abs. 1 Nr. 1 AMG verfolgen und dennoch arzneimittelspezifisch konzipiert sind, in den Anwendungsbereich des AMG aufzunehmen, da auch von diesen Mitteln Gefahren und Risiken ausgehen können, vor welchen das AMG die Bevölkerung schützen möchte. Als Beispiel werden hier Anästhetika, Aphrodisiaka, orale Kontrazeptiva oder Dopingmittel genannt. ${ }^{25}$ Bei allen diesen Mitteln handelt es sich zweifelsohne um Produkte, die physiologische Funktionen des Körpers beeinflussen, die jedoch nicht unter $\$ 2$ Abs. 1 Nr. 1 AMG fallen. Dass jedoch auch bei diesen Mitteln aufgrund ihrer Wirkung auf den Körper eine gesetzliche Regelung notwendig ist, erscheint nachvollziehbar. Der Einsatz von Tamoxifen als gentechnisches Werkzeug kann jedoch mit den Substanzen, für die die Regelung geschaffen wurde, nicht verglichen werden.

Dieses Ergebnis wird auch durch eine Betrachtung der allgemeinen Ziele des Arzneimittelgesetzes und ihre Gegenüberstellung mit den Zielen des Tierversuchsrechts untermauert.

20) Kügel/Müller/Hoffmann, Arzneimittelgesetz, 2. Aufl. 2016 , \$2 Rdnr. 88; KG, PharmR 2000, 339, 340; BVerwG, Urt. v. 25.7.2007 - 3 C 21/06, NVwZ 2008, 439, 440.

21) OVG Münster, Beschl. v. 23. 4.2012 - 13 B 127/12, NVwZ 2012 , 767, 768

22) OVG Münster, Beschl. v. 23.4.2012 - 13 B 127/12, NVwZ 2012, 767, 768; Rennert, NVwZ 2008, 1179, 1184

23) VG Köln, Urt. vom 20.3.2012 - 7 K 3169/11; VG Frankfurt (Oder), Beschl. vom 14.10.2011 - 4 L 191/11; Rehmann, AMG 3. Aufl., $₫ 2$ Rdnr. 2 und 6.

24) OVG Münster, Beschl. v. 23.4.2012 - 13 B 127/12, NVwZ 2012, 767, 768; EuGH, Urt. v. 15.11.2007 - C-319/05, ECLI:EU:C:2007:678 - Kommission/Deutschland, EuZW 2008, 56, Rdnr. 63-65; VG Köln, Urt. v. 20.3.2012 - 7 K 3169/11, Rdnr. $108 \mathrm{f}$.

25) Kügel/Müller/Hoffmann, Arzneimittelgesetz, 2. Aufl. 2016, $₫ 2$ Rdnr. 86. 
Das Tierversuchsrecht und das Arzneimittelrecht verfolgen unterschiedliche Zwecke. Während das Tierversuchsrecht Teil des Tierschutzrechts ist und den rechtlichen Rahmen für die Durchführung von Tierversuchen unter dem Gesichtspunkt des Tierschutzes definiert, ist das Ziel des Arzneimittelgesetzes die Sicherstellung einer ordnungsgemäßen Arzneimittelversorgung von Mensch und Tier sowie der Arzneimittelsicherheit (Qualität, Wirksamkeit und Unbedenklichkeit). Der von einem Gesetz verfolgte Zweck grenzt auch den durch den Gesetzgeber intendierten Anwendungsbereich dieses Gesetzes ein. Zu untersuchen ist daher, ob sich die Anwendungsbereiche des Tierschutzgesetzes und des Arzneimittelgesetzes gegenseitig ausschlieBen oder überschneiden, sodass das Arzneimittelgesetz auch auf tierexperimentelle Forschung Anwendung findet. Der auf den ersten Blick wahrnehmbare mögliche Überschneidungspunkt der beiden Anwendungsbereiche ist die Tatsache, dass beide Gesetze dem Schutz von Tieren dienen. Gleichwohl tun sie dies auf eine unterschiedliche Art und Weise. Das Arzneimittelgesetz will sicherstellen, dass eine ordnungsgemäße Versorgung der Tiere mit Arzneimitteln stattfindet ( $\$ 1 \mathrm{AMG}$ ), bezieht sich also auf die Stellung des Tieres als Patienten. Das Tierschutzgesetz und die darin enthaltenen Regelungen des Tierversuchsrechts sind hingegen ethisch motiviert und bezwecken den Schutz der Tiere vor Schmerzen, Leiden und Schäden, für die sich kein vernünftiger Grund nachweisen lässt ( $\$ 1$ TierSchG). Darüber hinaus ist die Aufgabe des Tierversuchsrechts, einen angemessenen Ausgleich zwischen dem schrankenlos gewährleisteten Grundrecht der Wissenschaftsfreiheit und dem Tierschutz zu schaffen.

Hinsichtlich der genauen Abgrenzung der Anwendungsbereiche der beiden Gesetze ist eine differenzierte Betrachtung geboten. Da auch zu Versuchszwecken gezüchtete und verwendete Tiere sich in der Stellung eines Patienten befinden können, ist in einer solchen Situation die Anwendbarkeit der Vorschriften des Arzneimittelgesetzes zu bejahen. Sobald es sich jedoch um die Anwendung von Arzneimitteln an Tieren handelt, die Teil des experimentellen Versuchsdesigns ist, erscheint der Ausschluss der Anwendbarkeit vom AMG sachgerecht. Eine erweiternde Auslegung des Funktionsarzneimittelbegriffes erscheint hier nicht geboten. Für diese Unterscheidung sprechen mehrere Gründe, insbesondere die systematische Auslegung der beiden Gesetze.

Die einzige explizite Regelung zu Versuchstieren, die das Arzneimittelgesetz trifft, bezieht sich auf klinische Prüfungen und Rückstandsprüfungen bei lebensmittelliefernden Tieren ( $\$ 59$ AMG). Es handelt sich hier um eine Ausnahme bezüglich der Anwendung von nicht zugelassenen Arzneimitteln für die o.g. klinischen bzw. Rückstandsprüfungen. Der Grund für die strenge Regulierung der Anwendung von Arzneimitteln bei lebensmittelliefernden Tieren ist der Verbraucherschutz. Insbesondere sollten mit der 16. AMGNovelle Maßnahmen ergriffen werden, um den Einsatz von Antibiotika in der Tierhaltung zu reduzieren. ${ }^{26}$ Aus der systematischen Stellung dieser Regelung kann geschlussfolgert werden, dass der Gesetzgeber im AMG lediglich den Bereich regeln wollte, in dem sich die Problematik der Durchführung von Tierversuchen und der Verbraucherschutz treffen. Es ist davon auszugehen, dass es dem Gesetzgeber bekannt war, dass auch im Bereich der Tierversuche Arzneimittel verwendet werden, dennoch wurde lediglich diese eine, sehr spezifische Regelung getroffen.

Für diese Schlussfolgerung spricht auch der Rechtsgedanke des $\$ 73$ Abs. 2 Nr. 2 AMG. $\$ 73$ AMG regelt ein grundsätzliches Verbringungsverbot für nicht zugelassene Arzneimittel. $\$ 73$ Abs. 2 Nr. 2 AMG bestimmt jedoch auch, dass dieses Verbot nicht gilt, wenn die Verbringung für den Eigenbedarf der Einrichtungen für Forschung und Wissenschaft bestimmt ist und die Substanz zu wissenschaftlichen Zwecken benötigt wird. Damit schafft das AMG eine Aus- nahme für die Bedürfnisse der Forschung. Diese Regelung könnte in dem systematischen Zusammenhang so gedeutet werden, dass es dem Gesetzgeber durchaus bewusst war, dass für die Forschung großzügigere Regelung gelten müssen, da es eben dem Wesen der Wissenschaft entspricht, Wirkungen von bestimmten Substanzen zu untersuchen oder durch ihren Einsatz bestimmte Effekte hervorzurufen. Dabei darf es keine Rolle spielen, ob diese Substanzen formell als Arzneimittel i.S.d. AMG anzusehen sind oder nicht, solange die Anwendung zu rein wissenschaftlichen Zwecken stattfindet und keine Gefährdung von Verbraucherinnen und Verbrauchern zu befürchten ist.

Diesen Ansatz bestätigt im Übrigen auch der Entwurf einer EU-Verordnung über Tierarzneimittel, ${ }^{27}$ mit dem angestrebt wird, einen einheitlichen und auf die Bedürfnisse des Veterinärsektors zugeschnittenen Rechtsrahmen zu schaffen und gleichzeitig die Gesundheit von Mensch und Tier zu schützen. Dieser Entwurf sieht im Art. 2 Nr. 4 Buchst. e vor, dass Tierarzneimittel, die für Versuche in Forschung und Entwicklung bestimmt sind, vom Anwendungsbereich dieser Verordnung ausgeschlossen sein sollen. Der EU-Gesetzgeber erkennt damit die Notwendigkeit an, für die Forschung Ausnahmen von den allgemeinen Vorschriften über den Umgang mit Arzneimitteln zu schaffen.

Die Schaffung der verschiedenen Kategorien der Arzneimittel beruht vor allem auf der Überlegung, eine bestmögliche und sichere Versorgung der Bevölkerung und der Tiere mit Arzneimitteln zu gewährleisten und gleichzeitig eine entsprechende Qualität, Wirksamkeit und Unbedenklichkeit sicherzustellen. So soll der Verbraucher vor den sich aus der Verwendung von Arzneimitteln ergebenden Gefahren geschützt werden. All diese Aspekte spielen jedoch bei der Anwendung von Tamoxifen als gentechnisches Werkzeug keine Rolle. Der Verbraucher kommt in der Regel mit Laborchemikalien nicht in Berührung, da die Abgabe solcher Substanzen durch besondere Vorschriften streng geregelt ist. Die Wissenschaftlerinnen und Wissenschaftler, die solche Substanzen in Versuchen einsetzen, sind in diesem Fall weniger schutzbedürftig, da sie sachkundig sind.

In der Praxis werden Sorgen geäußert, dass keine ausreichende Kontrolle über die Verabreichung von Substanzen wie Tamoxifen an Versuchstiere gewährleistet werden kann, wenn die Anwendbarkeit des Arzneimittelgesetzes auf diesen Sachverhalt verneint wird. Dabei ist jedoch zu beachten, dass die Anwendung von jeglichen Substanzen während eines Tierversuchs schon per se einer strengen Kontrolle unterliegt, da Tierversuche genehmigungspflichtig sind. Im Rahmen des Genehmigungsverfahrens müssen die Antragsteller detailliert darlegen, welche Eingriffe bzw. Behandlungen mit welchen Substanzen bei den Tieren durchgeführt werden. Die Behörde hat hier zum einen die Möglichkeit, entsprechende Auflagen zu erteilen, wenn sie der Meinung ist, dass die durch den Antragsteller anvisierte Art und Weise der Verabreichung die Tiere einem zu großen Risiko aussetzt. Zum anderen kann die Behörde durch vor Ort Kontrollen sicherstellen, dass die Substanzen sachgemäß eingesetzt werden. Dadurch wird den Belangen des Tierschutzes angemessen Rechnung getragen. Diese Meinung teilt auch das im Wege des Eilverfahrens mit der Problematik befasste Verwaltungsgericht Gera. In dem Beschluss vom 4. Mai 2018 ist das Gericht der Ansicht des Antragsgegners, die Anwendung des AMG würde der Sicherstellung des Tierwohls dienen, nicht gefolgt. Vielmehr hat das Gericht festgestellt, dass es nicht ersichtlich sei, inwiefern die Einhaltung der arzneimittelrechtlichen Vorschriften dem Wohl der Versuchstiere dienen kann. Aus dem

26) Heßhaus/Laber-Probst in: Kügel/Müller/Hofmann, Arzneimittelgesetz, 2. Aufl. 2016, §56a AMG, Rdnr. 2.

27) Vorschlag für eine Verordnung des Europäischen Parlaments und des Rates über Tierarzneimittel, COM (2014) 558 final. 
Tierschutzgesetz ließe sich diese Ansicht jedenfalls nicht begründen. ${ }^{28}$ Auch hält das Gericht Tamoxifen in Form der Laborchemikalie nicht für einen Stoff, der $\$ 2$ Abs. 1 Nr. 2a AMG unterfällt. Nach der Meinung des VG Gera kann die pharmakologische Wirkung alleine nicht ausreichen, um einen Stoff als Arzneimittel im Sinne des AMG zu klassifizieren. Das Gericht weist zu Recht daraufhin, dass bei der Auslegung des Arzneimittelbegriffes immer eine wertende Gesamtbetrachtung der Umstände anzustellen ist. Diese ergebe vorliegend, dass Tamoxifen als Laborchemikalie nicht als Arzneimittel anzusehen ist, da der Einsatz dieses Stoffes den Zweck des AMG nicht im Geringsten tangiert. ${ }^{29}$

Da im Bereich der Tierversuche der Verbraucherschutz i.d.R. keine Rolle spielt, besteht auch keine Notwendigkeit von zusätzlichen Einschränkungen der Anwendung bestimmter Substanzen im Tierversuch. Damit ist Tamoxifen in Form einer Laborchemikalie nicht als Arzneimittel i. S. d. Arzneimittelgesetzes sondern lediglich als eine pharmakologisch wirksame Substanz einzustufen.

\subsection{Regeln zum Bezug von Substanzen und Zubereitung der entsprechenden Injektionslösung bzw. des Futters durch die Forschenden}

Der Bezug von Tamoxifen und anderen Substanzen, die im Tierversuch eingesetzt werden, muss unter Beachtung der Vorschriften erfolgen, die den Handel mit solchen Substanzen und den Bezug von ihnen regeln, wie insbesondere das Chemikaliengesetz (ChemG) und die Chemikalien-Verbotsverordnung (ChemVerbotsV).

\section{Fazit}

Die Anwendung von pharmakologisch aktiven Substanzen an Tieren tangiert mehrere Rechtsbereiche. Deshalb ist eine eindeutige rechtliche Einordnung schwierig und muss stets im Hinblick auf den Sinn und Zweck der Gesetze erfolgen. Die Abwägung unter Berücksichtigung der Belange des Tierschutzes, der Wissenschaftsfreiheit und der Regelungsziele des AMG bezüglich des Umgangs mit Arzneimitteln gebietet es, die Anwendung von Tamoxifen als „gentechnisches Werkzeug“ zuzulassen, ohne dass diese
Art der Anwendung den Vorschriften des Arzneimittelrechts unterliegt.

Damit das Wohlbefinden der Tiere nicht unnötig beeinträchtigt wird, muss sichergestellt werden, dass die anzuwendende Injektionslösung unter Beachtung von hygienischen Vorschriften hergestellt wird. Dazu ist es jedoch nicht notwendig, dass die Anwendung von Tamoxifen bei Versuchstieren unter das Arzneimittelgesetz fällt. Die Herstellung der Lösung nach pharmazeutischen Grundsätzen sowie die Sicherstellung, dass die Injektion nach allen Regeln der Kunst durch qualifiziertes Personal durchgeführt wird, kann im Rahmen des Genehmigungsverfahrens kontrolliert werden. Die zuständige Behörde kann bezüglich der Herstellung der Injektionslösung entsprechende Auflagen erteilen, um die Einhaltung entsprechender Standards zu gewährleisten. Ggf. kann die Behörde auch die Vorlage von entsprechenden Standard Operating Procedures (SOPs) oder anderen Verfahrensanweisungen anordnen. Auf diese Art und Weise kann ein angemessener Ausgleich zwischen den verschiedenen relevanten Belangen erreicht werden.

Open Access. Dieser Artikel wird unter der Creative Commons Namensnennung 4.0 International Lizenz veröffentlicht, welche die Nutzung, Vervielfältigung, Bearbeitung, Verbreitung und Wiedergabe in jeglichem Medium und Format erlaubt, sofern Sie den/die ursprünglichen Autor(en) und die Quelle ordnungsgemäß nennen, einen Link zur Creative Commons Lizenz beifügen und angeben, ob Änderungen vorgenommen wurden.

Die in diesem Artikel enthaltenen Bilder und sonstiges Drittmaterial unterliegen ebenfalls der genannten Creative Commons Lizenz, sofern sich aus der Abbildungslegende nichts anderes ergibt. Sofern das betreffende Material nicht unter der genannten Creative Commons Lizenz steht und die betreffende Handlung nicht nach gesetzlichen Vorschriften erlaubt ist, ist für die oben aufgeführten Weiterverwendungen des Materials die Einwilligung des jeweiligen Rechteinhabers einzuholen.

Weitere Details zur Lizenz entnehmen Sie bitte der Lizenzinformation auf http://creativecommons.org/licenses/by/4.0/deed.de.

28) VG Gera, Beschl. v. 4. 5.2018 - 2 E 383/18 Ge, S. 8.

29) VG Gera, Beschl. v. 4. 5.2018 - 2 E $383 / 18$ Ge, S. 9 f. 